\title{
Mentorship in anesthesia: how little we know
}

\author{
Alana M. Flexman, MD • Adrian W. Gelb, MBChB
}

Received: 21 November 2011/Accepted: 14 December 2011/Published online: 28 December 2011

(C) Canadian Anesthesiologists' Society 2011

Mentorship has been shown to be an important factor during career progression in the spheres of both medicine and business. ${ }^{1-3}$ While mentorship is frequently related to success in research, it likely has an impact on many aspects of medical practice, including clinical and educational skills. Mentorship has been defined as a relationship in which a person with useful experience, knowledge, skills, and/or wisdom offers advice, information, guidance, support, or opportunity to another for that individual's professional development. "Mentees" are typically more junior than their mentors, and they may have several mentors for various aspects of their career development. Mentors may offer general career guidance, specialized knowledge, research advice, and networking opportunities. Mentorship may result from formal programs or through informal relationships. Formal mentorship is facilitated through programs that provide matching of mentor-mentee pairs, while informal mentorship exists when both parties voluntarily establish the relationship. With both types of mentorship, the mentor and mentee should be compatible in personality, interests, and goals. ${ }^{5}$

The current literature strongly supports the benefits of mentorship in medicine. Mentored physicians have been

\footnotetext{
A. M. Flexman, MD

Department of Anesthesiology, Pharmacology and Therapeutics, University of British Columbia Vancouver General Hospital,

Vancouver, BC, Canada
}

A. W. Gelb, MBChB ( $₫)$

Department of Anesthesia and Perioperative Care, University of California San Francisco, 521 Parnassus Avenue, Room C-450, San Francisco, CA 94143-0648, USA shown to have increased career satisfaction and greater confidence as educators. ${ }^{1}$ In addition, physicians who are mentored are more successful in acquiring promotions, publishing their work, and obtaining peer-reviewed grant funding. ${ }^{1-3,6-8}$ Having an identified mentor may influence career choice, as multiple studies have suggested that physicians who are mentored are more liable to pursue an academic career. ${ }^{1}$ Finally, mentored physicians may be more likely to mentor others and thus impart the benefits of mentorship to others. ${ }^{2}$

Mentors often wonder if any benefit accrues to them. Providing mentorship is associated with increased personal satisfaction and professional stimulation. ${ }^{9,10}$ If the mentee is successful, the mentor may benefit from increased productivity and professional recognition. ${ }^{9}$ Increasingly, providing mentorship is considered during assessment for academic promotion and thus may directly influence the mentor's career progression. ${ }^{5}$

Although mentorship is beneficial to both mentors and mentees, several barriers to achieving mentorship have been identified in the literature. Two common problems in establishing mentorship are a lack of skilled mentors and insufficient time available for mentoring. ${ }^{5,9,11}$ The establishment of mentorship training programs and regional or national mentorship networks are potential ways to augment the mentorship pool. Access to protected non-clinical time for mentorship is a potential solution to the time constraints on both parties. ${ }^{5}$ Additionally, certain mentee characteristics may be associated with obstacles to obtaining mentorship. For example, some studies have shown that women and minority groups are less likely to have an identified mentor. Female or minority mentors may have increased understanding of the issues faced by these groups and may be even more difficult to identify. ${ }^{10-12}$ Formal mentorship programs targeting these populations 
may improve access to mentorship. ${ }^{5}$ Finally, physicians whose focus is clinical medicine or education are less likely to be mentored than those who are primarily research-focused. ${ }^{6}$ This group, which makes up the largest proportion of clinical departments, would likely benefit from mentorship in educational and clinical performance as well as administrative leadership.

Despite the increasing acceptance of mentorship as a critical part of career development, little is known about mentorship in anesthesiology. Given the close relationship between mentorship and academic productivity, the lack of data is worrisome. The limited growth in peerreviewed grant funding and the projected increased clinical demands in anesthesiology now threaten our status as a respected discipline in academic medicine. ${ }^{13}$ Without consistent and high-quality mentorship, our growth as academic and healthcare leaders may continue to be restricted. ${ }^{13}$ Finally, a recent editorial further highlights the potential role of mentorship in preventing ethical misconduct. ${ }^{14}$ The mentor has an important role in promoting integrity by teaching, clarifying, and modelling the rules of ethical conduct. ${ }^{14}$ All of the above are compelling reasons to promote mentorship in anesthesiology, yet little is known to date. Potential areas of research are outlined below.

The first step is to identify the prevalence of mentorship in anesthesiology. Currently, the prevalence of both formal and informal mentorship among both trainees and faculty in anesthesiology is unknown. While some centres have formal mentorship programs in place, the overall prevalence of such programs is unknown, and we do not know if there is a desire for such programs. At a minimum, this information will highlight potential areas where access to mentorship needs to be improved.

Although barriers to mentorship have been identified in other specialties, those specific to anesthesiology are unknown. These factors may relate to the academic or clinical centre itself, such as size or geographic location. Similar to other specialties, certain physician groups may have difficulty obtaining mentorship and should be identified. For example, although the low number of female anesthesiologists pursuing academic careers is not unique to our specialty, ${ }^{15}$ the issues in anesthesiology facing this group may differ, and specific strategies may be required to address them. In addition, time constraints may be a significant problem in anesthesiology given that clinical practice in anesthesiology is often intense and isolating. Similar to other specialties, access to skilled mentors is likely a major obstacle. The lack of emphasis on mentorship may hamper the development of academic anesthesiology departments. Individuals with an early interest in research or education but without access to the guidance and advice of a mentor may likely represent untapped potential in academic anesthesiology.

The benefits of mentorship on academic career development in anesthesiology should be clarified. The impact of mentorship on academic productivity, promotion, and retention of trainees and faculty is important to document, particularly during the implementation of mentorship programs. Evidence of the advantages of mentorship in anesthesiology will further encourage departments to facilitate these relationships and reinforce their commitment to mentorship. This research should focus not only on research-oriented academic physicians but also on academic physicians with an emphasis on education and clinical work.

Finally, further investigation is required into the optimal structure of mentorship programs in anesthesiology. For example, mentorship networks on a national or international scale may improve access to high-quality mentorship, particularly amongst those identified as having difficulty accessing mentorship. Given the growing clinical demands in most anesthesiology departments, it is likely that access to research mentorship is limited. While the Foundation for Anesthesia Education and Research recognizes members of its mentoring academy, few resources are available to those without mentors. Finally, training programs for mentors and methods to assess mentorship quality need to be developed in anesthesiology.

In summary, mentorship is important to academic career development and should be considered an essential component of anesthesiology departments, yet few data exist on this topic. Further research is required to determine the prevalence of mentorship programs and the impact of such programs on the recruitment and retention of faculty and trainees. Groups with difficulty obtaining mentorship in anesthesiology should be identified and potential solutions to these obstacles should be determined, including the need for targeted mentorship programs. Research into the benefits of mentorship should focus not only on research productivity but also on educational performance and clinical excellence. The recruitment and retention of academic anesthesiology faculty is an issue of critical importance to our specialty at the present time. Given the positive effect of mentorship on career choice, satisfaction, and productivity in other disciplines, mentorship should be a core component of anesthesiology. Overall, mentorship is an exciting area of future research that may significantly impact the growth and development of the specialty of anesthesiology. 


\section{Mentorat en anesthésie: nous en savons si peu...}

Nous savons maintenant que le mentorat est un facteur important dans l'évolution d'une carrière, dans la sphère des affaires, comme dans celle de la médecine. Alors que le mentorat est fréquemment lié à la réussite de la recherche, il a probablement aussi un impact sur de nombreux aspects de l'exercice de la médecine, y compris sur les compétences cliniques et formatrices. Le mentorat a été défini comme une relation au cours de laquelle une personne riche d'une expérience, de savoirs, de compétences ou de raisonnements utiles offre des conseils, de l'information, un accompagnement, un soutien ou une chance à une autre personne pour qu'elle acquière ce perfectionnement professionnel personnel. Les « mentorés » sont habituellement plus jeunes que leurs mentors et ils peuvent aussi avoir plusieurs mentors couvrant différents aspects de leur développement professionnel. Les mentors peuvent offrir une orientation générale dans la carrière, un savoir spécialisé, un conseil pour une recherche et des possibilités de réseautage. Le mentorat peut être le résultat de programmes officiels ou de rapports informels. L'organisation formelle du mentorat peut être facilitée par des programmes procurant des appariements de paires mentors-mentorés tandis qu'un mentorat informel existe lorsque les deux parties établissent volontairement une telle relation. Dans les deux types de mentorat, il doit y avoir une compatibilité de personnalités, d'intérêts et d'objectifs entre le mentor et le mentoré. ${ }^{5}$

La littérature actuelle fait nettement ressortir les avantages du mentorat en médecine. Les médecins mentorés ont démontré une plus grande satisfaction dans leur carrière et une plus grande confiance en eux en tant qu'éducateurs. ${ }^{1}$ De plus, les médecins qui sont mentorés réussissent davantage à obtenir des promotions, à publier leurs travaux et à obtenir un financement grâce à des subventions attribuées par des comités de sélection. ${ }^{1-3,6-8}$ Avoir un mentor connu peut influencer un choix de carrière: de multiples études ont suggéré que les médecins qui avaient un mentor avaient plus de chances de poursuivre une carrière universitaire. ${ }^{1}$ Enfin, les médecins mentorés agiront plus probablement à leur tour comme mentors, transmettant ainsi les avantages du mentorat à d'autres. ${ }^{2}$

Les mentors se demandent souvent quels avantages ils tirent de leur activité. Assurer un mentorat est associé à une plus grande satisfaction personnelle et à une plus grande stimulation professionnelle. ${ }^{9,10} \mathrm{Si}$ le mentoré réussit, le mentor pourra bénéficier d'une meilleure productivité et d'une reconnaissance professionnelle.' De plus en plus, l'évaluation en vue d'une promotion universitaire prend en compte une activité de mentorat, ce qui peut donc avoir une influence directe sur l'évolution de la carrière du mentor. ${ }^{5}$

Bien que le mentorat profite à la fois aux mentors et aux mentorés, plusieurs obstacles à la réalisation du mentorat ont été identifiés dans la littérature. Deux problèmes fréquents à la création d'un mentorat est le manque de mentors compétents et un manque de temps disponible à y consacrer. $^{5,9,11}$ La mise en place de programmes de formation au mentorat ainsi que les réseaux régionaux et nationaux de mentorat sont des moyens potentiels d'augmenter le nombre de mentors. Disposer de temps protégé non clinique réservé au mentorat peut être une solution aux contraintes de temps des deux parties concernées. 5 De plus, certaines caractéristiques des mentorés peuvent être associées à des obstacles pour l'obtention d'un mentorat. Certaines études ont, par exemple, montré que les femmes et les groupes minoritaires avaient moins de chances d'avoir un mentor identifié. Les mentors femmes ou issus des minorités pourraient mieux comprendre les problèmes rencontrés par ces groupes et pourraient être encore plus difficiles à identifier. $^{10-12}$ Des programmes officiels de mentorat ciblant ces populations pourraient améliorer l'accès au mentorat. ${ }^{5}$ Enfin, les médecins s'orientant vers la médecine clinique ou l'éducation ont une moindre probabilité d'avoir un mentor que ceux s'orientant principalement vers la recherche. 6 Ce groupe, qui constitue l'essentiel des effectifs des départements cliniques, bénéficierait probablement d'un mentorat en matière d'éducation, de performances cliniques et aussi de leadership administratif.

En dépit de l'acceptation croissante du mentorat comme élément essentiel d'une évolution de carrière, on ne sait que peu de choses sur le mentorat en anesthésiologie. Compte tenu des liens étroits existant entre le mentorat et la productivité universitaire, ce manque de données est inquiétant. La croissance limitée des subventions attribuées par des comités de sélection et l'augmentation croissante prévue des demandes cliniques en anesthésiologie menacent maintenant notre statut en tant que discipline universitaire respectée. ${ }^{13}$ Sans un mentorat constant et de haut niveau, notre progression en tant que chefs de file au niveau universitaire et dans le domaine de la santé pourrait continuer à se resserrer. ${ }^{13}$ Par ailleurs, un éditorial récent a souligné le rôle potentiel du mentorat dans la prévention de fautes éthiques. ${ }^{14}$ Le mentor joue un rôle important en faisant par son enseignement, la promotion de l'intégrité, en clarifiant et en modelant les règles de comportement éthique. ${ }^{14}$ Toutes les raisons ci-dessus sont des motifs convaincants de promotion du mentorat en anesthésiologie, bien que peu connues à ce jour. Les domaines potentiels de recherche sont discutés ci-dessous.

La première étape consiste à identifier la prévalence du mentorat en anesthésiologie. Actuellement, la prévalence 
des deux formes de mentorat (officiel et informel) en anesthésiologie parmi les médecins en formation et les enseignants est inconnue. Alors que quelques centres ont institué des programmes officiels de mentorat, la prévalence globale de tels programmes est inconnue et nous ignorons s'il existe une demande pour de tels programmes. Au minimum, cette information éclairera les domaines potentiels dans lesquels l'accès au mentorat a besoin d'être amélioré.

Même si des obstacles au mentorat ont été identifiés dans d'autres spécialités, ceux qui sont propres à l'anesthésiologie sont inconnus. Il pourrait s'agir de facteurs propres au centre universitaire ou clinique, proprement dit, comme sa taille ou sa situation géographique. Comme dans d'autres spécialités, certains groupes de médecins peuvent avoir du mal à obtenir un mentorat et ils doivent être identifiés. Même si, par exemple, le fait que peu de femmes anesthésiologistes poursuivent une carrière universitaire n'est pas propre à notre spécialité, ${ }^{15}$ les problèmes auxquels sont confrontés ce groupe peuvent être différents et des stratégies spécifiques pourraient être nécessaires pour les résoudre. De plus, les contraintes de temps peuvent être un problème significatif en anesthésiologie, considérant que la pratique clinique de cette spécialité est souvent intense et constitue un facteur d'isolement. Comme dans d'autres spécialités, l'accès à des mentors chevronnés risque d'être un obstacle majeur. L'absence d'insistance sur le mentorat peut entraver le développement des départements universitaires d'anesthésiologie. Des personnes manifestant un intérêt précoce pour la recherche ou l'éducation, mais dépourvus des conseils et de l'orientation apportés par un mentor représentent probablement un potentiel inexploité pour l'anesthésiologie universitaire.

Les avantages du mentorat sur l'évolution d'une carrière universitaire en anesthésiologie doivent être clarifiés. Il est important de documenter l'impact du mentorat sur la productivité et la promotion universitaires, sur la rétention des médecins en formation ainsi que des enseignants, notamment au cours de la mise en ouvre des programmes de mentorat. La preuve des avantages du mentorat en anesthésiologie continuera à encourager les départements à rendre ces rapports plus faciles et à renforcer leur engagement dans cette voie. Cette recherche doit se concentrer non seulement sur les médecins universitaires ayant une orientation vers la recherche, mais aussi sur les médecins universitaires qui se consacrent surtout à l'enseignement et au travail clinique.

Enfin, des études complémentaires sur la structure optimale des programmes de mentorat en anesthésiologie sont nécessaires. Des réseaux de mentorat à l'échelle nationale ou internationale pourraient améliorer l'accès à un mentorat de haute qualité, en particulier pour ceux qui auront été identifiés comme ayant des problèmes d'accès à ce mentorat. Compte tenu des exigences cliniques croissantes dans la plupart des départements d'anesthésiologie, il est probable que l'offre de mentorat de recherche soit limité. Bien que la Fondation pour l'éducation et la recherche en anesthésie (Foundation for Anesthesia Education and Research) admette des mentors comme membres, il y a peu de ressources disponibles pour ceux qui ne disposent pas d'un mentor. Enfin, des programmes de formation des mentors et des méthodes d'évaluation de la qualité du mentorat doivent être élaborés en anesthésiologie.

En résumé, le mentorat est important pour l'évolution d'une carrière universitaire et doit être vu comme un élément essentiel dans les départements d'anesthésiologie; il existe cependant peu de données sur ce sujet. Des recherches complémentaires sont nécessaires pour connaître la prévalence des programmes de mentorat et leur impact sur le recrutement et la rétention des médecins en formation et des enseignants universitaires. Les groupes ayant du mal à accéder au mentorat en anesthésiologie doivent être identifiés de même que les solutions possibles à ces difficultés, y compris le besoin de programmes de mentorat ciblés. Une étude sur les avantages du mentorat ne doit pas seulement se concentrer sur la productivité de la recherche, mais aussi sur la performance en enseignement et l'excellence en clinique. À l'heure actuelle, le recrutement et la rétention des enseignants en anesthésiologie sont une question absolument essentielle pour notre spécialité. Compte tenu, dans d'autres disciplines, de l'effet positif du mentorat sur le choix de carrière, la satisfaction qu'elle procure et la productivité, le mentorat doit être une composante centrale en anesthésiologie. Globalement, le mentorat est un domaine fascinant de recherche d'avenir qui pourra avoir un impact significatif sur la croissance et le développement de l'anesthésiologie.

Competing interests None declared.

\section{References}

1. Sambunjak D, Straus SE, Marusic A. Mentoring in academic medicine: a systematic review. JAMA 2006; 296: 1103-15.

2. Steiner JF, Curtis P, Lanphear BP, Vu KO, Main DS. Assessing the role of influential mentors in the research development of primary care fellows. Acad Med 2004; 79: 865-72.

3. Steiner $J F$, Lanphear BP, Curtis $P, V u K O$. Indicators of early research productivity among primary care fellows. J Gen Intern Med 2002; 17: 845-51.

4. Berk RA, Berg J, Mortimer R, Walton-Moss B, Yeo TP. Measuring the effectiveness of faculty mentoring relationships. Acad Med 2005; 80: 66-71.

5. Flexman AM, Gelb AW. Mentorship in anesthesia. Curr Opin Anaesthesiol 2011; 24: 676-81. 
6. Feldman MD, Arean PA, Marshall SJ, Lovett M, O'Sullivan P. Does mentoring matter: results from a survey of faculty mentees at a large health sciences university. Med Educ Online 2010; DOI:10.3402/meo.v15i0.5063.

7. Orandi BJ, Blackburn S, Henke PK. Surgical mentors' and mentees' productivity from 1993 to 2006. Am J Surg 2011; 201: 260-5.

8. Ramanan RA, Taylor WC, Davis RB, Phillips RS. Mentoring matters. Mentoring and career preparation in internal medicine residency training. J Gen Intern Med 2006; 21: 340-5.

9. Keyser DJ, Lakoski JM, Lara-Cinisomo S, et al. Advancing institutional efforts to support research mentorship: a conceptual framework and self-assessment tool. Acad Med 2008; 83: 217-25.

10. Kosoko-Lasaki O, Sonnino RE, Voytko ML. Mentoring for women and underrepresented minority faculty and students: experience at two institutions of higher education. J Natl Med Assoc 2006; 98: 1449-59.
11. Straus SE, Chatur F, Taylor M. Issues in the mentor-mentee relationship in academic medicine: a qualitative study. Acad Med 2009; 84: 135-9.

12. Levine RB, Lin F, Kern DE, Wright SM, Carrese J. Stories from early-career women physicians who have left academic medicine: a qualitative study at a single institution. Acad Med 2011; 86: $752-8$.

13. Schwinn DA, Balser JR. Anesthesiology physician scientists in academic medicine: a wake-up call. Anesthesiology 2006; 104: 170-8.

14. Ochroch EA, Eckenhoff $R G$. The role of mentoring in aiding academic integrity. Anesth Analg 2011; 112: 732-4.

15. Wong CA, Stock MC. The status of women in academic anesthesiology: a progress report. Anesth Analg 2008; 107: 178-84. 